\title{
Assembling insight
}

\section{Model colloidal systems are a testbed for understanding aspects of the organization of matter.}

Colloidal particles are the ultimate building blocks for assembly from the bottom-up. They can be synthesized in many shapes and sizes, and the interactions between them designed and adjusted - for example, by decorating the particles with ligands, by coating their surface with metals or polymers, or by changing the make-up, the $\mathrm{pH}$, the density or the temperature of the solvent they are embedded in. Colloidal systems are also the simulator's dream, if only because many of their models and findings have been later realized and corroborated in the laboratory. Moreover, colloids are often big enough and move sufficiently slowly to be individually tracked with optical microscopes; hence, one can easily study their dynamics and arrangement into ordered or amorphous aggregates. Many consider model colloidal systems to be 'big atoms', much easier to play with than the real ones. As such, colloids can mimic some of the behaviour of nanocrystals, proteins, liquid crystals, vesicles, bacteria, vibrated macroscopic granules and many other particulate matter. Beyond mimicking other systems, model colloids shine at providing new understanding, often of the mechanistic variety. This is exemplified by the content included in this focus issue on colloidal matter.

On page 101, Yilong Han and colleagues show that, for hard colloids, the transformation between two simple lattices (a square lattice that becomes a hexagonal one) occurs via the nucleation of the hexagonal lattice within a liquid nucleus formed in the 'parent' square crystal. Most known transformations between crystals occur through diffusionless (martensitic) transitions, yet Han and colleagues suggest that the indirect 'nucleus-in-anucleus' pathway, where the intermediate liquid shields the crystals from forming the typically more costly crystal/crystal interfaces, could be a general mechanism, even in atomic and molecular systems. In an accompanying News \& Views piece on page 15, Eduardo Sanz and Chantal Valeriani emphasize that, in addition to the formation of liquid/crystal interfaces (rather than interfaces between crystals) being more favourable, the liquid must be thermodynamically more stable than the parent crystal.

Thermodynamic stability in colloidal systems can be designed by tweaking the interactions between the colloids. For

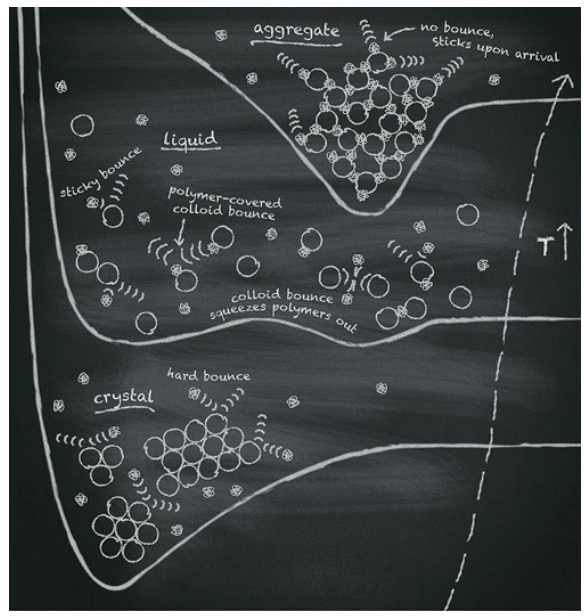

Figure 1 | In a mixture of colloids (empty circles) and polymers (filled circles), the liquid can solidify both on increasing or decreasing temperature, forming a disordered aggregate or a crystal, respectively. Image courtesy of Mirjam Leunissen, Dutch Data Design (http://dutchdatadesign.nl).

example, the addition of polymer to a dense colloidal dispersion increases the stability of colloidal aggregates because the polymer mediates an effective attractive force between the colloids. The origin of such a force is purely entropic: the volume accessible to the polymer increases if the colloids and the polymer separate into two phases, because in mixed configurations the polymer is excluded from the gaps between colloidal particles (when the gaps are smaller than the diameter of the polymer particles). And as Lang Feng, Paul Chaikin and collaborators show on page 61 , if the polymer partially adsorbs on the colloids, the colloid-polymer dispersion can solidify both when heated or cooled (Fig. 1). Steve Granick and co-authors argue in a News \& Views on page 17 that such temperature-switchable systems could be used to make colloidal assemblies with sequentially programmed order.

Although excluded-volume interactions have long been studied, only in the past few years have they been harnessed to make materials with designed order. In fact, entropy can favour the formation of crystals, and even crystalline clusters with icosahedral order (the local order in liquids), as Marjolein Dijkstra and colleagues demonstrate on page 56. In a Commentary on page 9, Daan Frenkel explains why entropy can indeed lead to order, and stresses the importance of understanding entropy in colloidal self-assembly, in particular in the design of materials with addressable complexity - that is, materials self-assembled from many components, each of them occupying a specified position. Most designs of self-assembled structures are based on the 'puzzle' approach, where components diffuse freely to reach specific local configurations rather than be constrained to a long thread that can fold. Yet, as Ludovico Cademartiri and Kyle Bishop discuss in a Commentary on page 2, designing colloidal systems that 'fold' into a specific shape (as with proteins) may offer scalability and kinetic advantages.

When freed from experimental constraints, the design of particle-particle interactions can lead to unexpected findings. Michael Engel, Sharon Glotzer and coauthors report on page 109 the self-assembly of the first icosahedral quasicrystal from a one-component system of particles interacting via forces that oscillate with interparticle distance. In a News \& Views on page 18, Marc de Boissieu discusses how the authors' simulated system should further our understanding of the formation of icosahedral quasicrystals in intermetallic alloys. Indeed, computer simulations are sometimes the only available route to gather mechanistic understanding of the behaviour of complex systems. By using experiments and simulations in a complementary manner, Michael Solomon, Sharon Glotzer and colleagues describe on page 117 the assembly of colloidal ellipsoids into microscopic fibres, and their extension and contraction when an electric field is applied. Eric Furst notes in a News \& Views on page 19 that simulations could be further used to guide experiments in the optimization of the extensibility of the fibrillar aggregates.

Interactions between colloidal particles, and thus their assembly products, can also be affected by electromagnetic fields. Nicholas Kotov and colleagues show on page 66 that, unexpectedly, circularly polarized light induces the formation of an excess of self-assembled chiral ribbons of semiconductor nanoparticles with the same handedness (left or right) as that of the light. Yet, as Bart Kahr points out in a News \& Views on page 21, pinning down the actual mechanism among the various possibilities is mostly a matter of assembling further insight. 